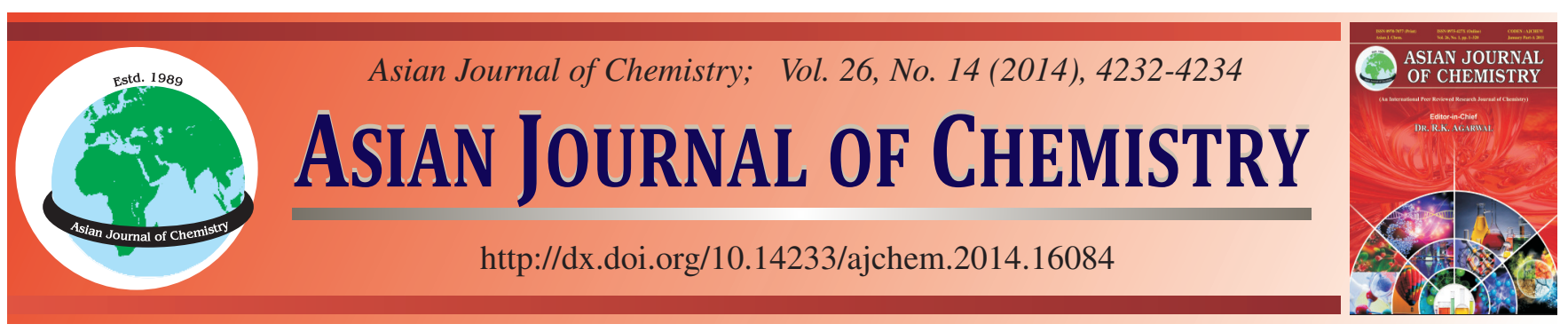

\title{
Hydroxypropyl- $\beta$-Cyclodextrin Functionalized Graphene Oxide Nanospheres as Unmodified Paclitaxel Carriers
}

\author{
Yi He, Danfeng Chen and GuOMin Xiao*
}

School of Chemistry and Chemical Engineering, Southeast University, Nanjing 211189, Jiangsu Province, P.R. China

*Corresponding author: Tel: +86 25 52090612; E-mail: xiaogm@seu.edu.cn

Received: 22 July 2013;

Accepted: 11 November 2013;

Published online: 5 July 2014;

AJC-15448

This work reports a novel type of electrosprayed graphene oxide nanospheres consisting of gelatin and hydroxypropyl- $\beta$-cyclodextrin functionalized graphene oxide sheets to support water insoluble anticancer drug paclitaxel. The drug loading content and drug loading efficiency were as high as $14.9 \mu \mathrm{g} / \mathrm{mg}$ and $59.5 \%$, respectively. The slow and smooth in-vitro release profiles of paclitaxel were assigned to the strong interaction between graphene oxide sheets and the hydroxypropyl- $\beta$-cyclodextrin molecules, in addition to the encapsulation of electrosprayed graphene oxide nanospheres.

Keywords: Nanocomposite, Graphene Oxide, Electrospray.

\section{INTRODUCTION}

Poor solubility of water-insoluble anti-cancer drugs is a critical limitation to oral absorption therefore has hampered their use for disease treatments. For instance, paclitaxel (PTX) has to be dispersed in toxic solvent, such as Cremephor E, inducing serious anaphylaxis or other dangerous inflammations ${ }^{1-5}$, which is a quite urgent issue to address.

The graphene oxide (GO) has rich oxygenated groups at both surfaces and edges, allowing sufficient active sites for supporting anticancer drugs or biocompatible molecules, such as cyclodextrins $(\mathrm{CDs})^{6-8}$. Hydroxypropyl- $\beta$-cyclodextrin is a $\beta$-cyclodextrin derivative and is made up of cyclic oligosaccharides consisting of $\alpha(1,4)$-linked glucopyranose units creating a truncated cone and hydrophobic cavity molecular structure ${ }^{9}$. Such unique molecular shape generates "host-guest" inclusion complexes with varied small molecules, which form the reversible inclusion complexes with the cavity, thus dramatically increase their water solubility and stabilities ${ }^{10}$.

Herein, we designed a new type of graphene oxide nanospheres as unmodified paclitaxel carriers (Scheme-I). The

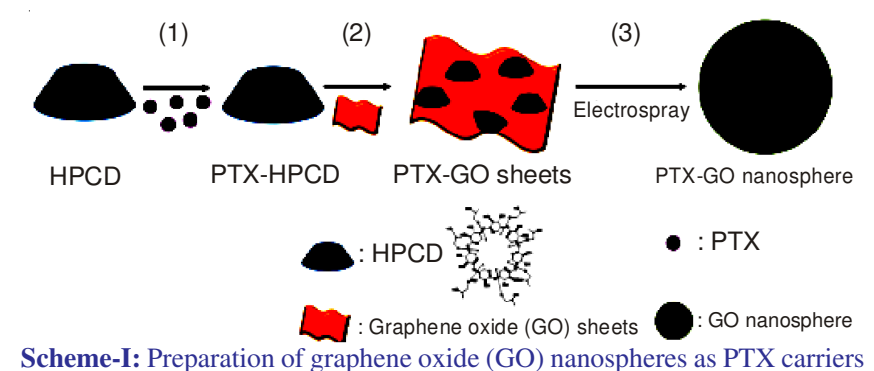

paclitaxel were (1) firstly included in hydroxypropyl- $\beta$ cyclodextrin cavities, (2) then were anchored on the graphene oxide sheets surface via covalent interaction, generating PTXGO sheets. (3) The functionalized graphene oxide sheets were homogeneously mixed with gelatin solution and electrospray to form graphene oxide nanospheres in uniform sizes.

\section{EXPERIMENTAL}

All chemicals were obtained from Sinopharm Chemical Reagent Co., Ltd except for the paclitaxel from Aladdin Chemical Reagent Co., Ltd.

Paclitaxel (150 mg) was dissolved in $30 \mathrm{~mL}$ of ethanol, followed by adding $0.5 \mathrm{~g}$ of hydroxypropyl- $\beta$-cyclodextrin. The mixed solution was magnetically stirred at room temperature for one day and dried overnight in vacuum. $10 \mathrm{mg}$ of resulted white powders were dispersed in $5 \mathrm{~mL}$ of distilled water and centrifuged to remove the excess paclitaxel left at centrifuge tube bottom. $10 \mathrm{~mL}$ of $1 \mathrm{mg} / \mathrm{mL}$ graphene oxide colloid prepared by Hummers method ${ }^{11}$, were added into the aqueous solution of PTX-HPDC followed by the incubation at room temperature for $24 \mathrm{~h}$ then centrifuged to remove the unanchored PTX-HPDC. Finally the PTX-GO oxide sheets were freeze-dried overnight.

$10 \mathrm{mg}$ of PTX-GO oxide sheets and $500 \mathrm{mg}$ of gelatin were dissolved in $50 \mathrm{~mL}$ of acetic acid then mildly stirred for $10 \mathrm{~h}$. The solution was loaded into a plastic syringe tipped a spinneret at bottom. A $10 \mathrm{kV}$ of direct voltage electrified the spinneret and the graphene oxide nanospheres were electrosprayed at a feeding rate of $0.5 \mathrm{~mL} / \mathrm{h}$. 
The amounts of paclitaxel were tested by high performance liquid chromatography (HPLC, THERMO ELECTRONLCQAD-60000) with UV detection at $227 \mathrm{~nm}$ using a 1/1 (v/v) mixture of acetonitrile and water as the mobile phase. The drug loading efficiency (DLE) and drug loading concentration (DLC) were calculated by the following equations:

$\begin{gathered}\begin{array}{c}\text { Drug loading } \\ \text { efficiency } \\ (\text { wt. \%) }\end{array} \\ \begin{array}{c}\text { Drug loading } \\ \text { content } \\ (\text { wt. } \%)\end{array}\end{gathered}=\frac{\begin{array}{c}\text { Weight of loaded } \\ \text { paclitaxel in carriers }\end{array}}{\text { Weight of paclitaxel in feed }} \times 100 \%$

PTX-GO nanospheres $(100 \mathrm{mg}$ ) were sealed in a dialysis bag $(\mathrm{Mmwco}=3.5 \mathrm{kDa})$ and incubated with $50 \mathrm{~mL}$ of PBS $(\mathrm{pH}=7.4)$ at $37^{\circ} \mathrm{C}$. At a different given interval time, $2 \mathrm{~mL}$ of PBS solution was taken out and the same volume of fresh PBS solution was retrieved in the same time.

\section{RESULTS AND DISCUSSION}

Atomic force microscopy (AFM, MFP-3D-SA, Asylum Research) image (Fig. 1A) of graphene oxide sheets exhibits their lateral sizes are in the range of several hundreds to thousand nanometers. These sheets are highly flexible and tend to wrinkle as a result of minimization of their surface energies. And the wrinkle is also observed under transmission electron microscopy (TEM, Tecnai G2 T20, FEI) (Fig. 1B). Their high flexibilities would facilitate their formation of nanospheres during electrospraying. The heights of the crossed the graphene oxide sheets are $7.12 \AA$ (red), $6.38 \AA$ (blue) and $6.51 \AA$ (green), respectively, illustrating their single-layer thicknesses ${ }^{12,13}$.
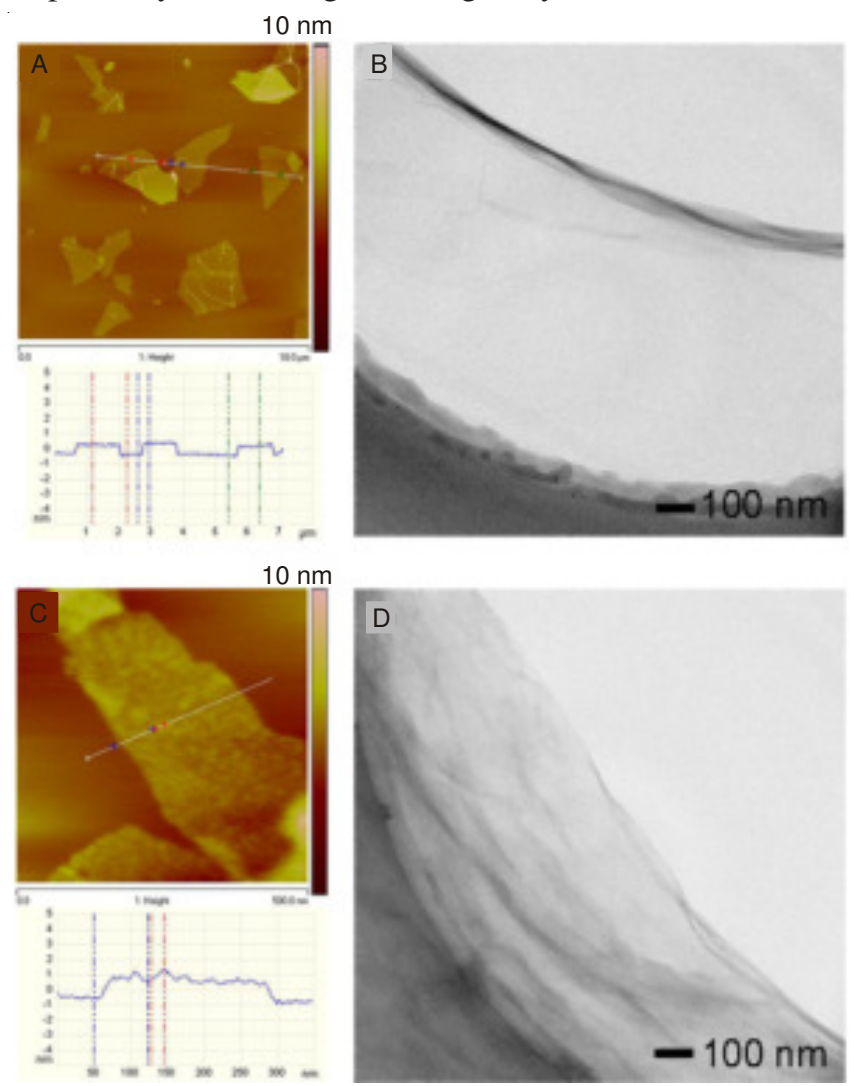

Fig. 1. AFM (left) and TEM (right) images of (A, B) graphene oxide sheets and $(\mathrm{C}, \mathrm{D}) \mathrm{PTX}-\mathrm{GO}$
The paclitaxel was included in the hydroxypropyl- $\beta$ cyclodextrin (HPCD) cavity by "host-guest" mechanism ${ }^{10}$. Its drug loading content and drug loading efficiency were as high as $243 \mu \mathrm{g} / \mathrm{mg}$ and $81.0 \%$, respectively. The PTX-HPCD was ready to anchor graphene oxide sheets in water due to ester bonds, hydrogen bonding, $\pi-\pi$ stacking and strong vander Walls interaction, generating PTX-GO sheets $^{7,8,14}$. Fig. 1C shows the AFM image of one piece of PTX-GO sheet and its height profile. Their surface becomes uneven and the thickness increases to $1.25 \mathrm{~nm}$ accordingly. The position between two red lines presents a height of $6.04 \AA$ and responds to the PTXHPCD molecules ${ }^{14}$. Furthermore, the smooth height profile on PTX-GO surface indicates the uniform PTX-HPCD dispersion at graphene oxide surface and the absence of aggregation. The TEM in Fig. 1D also demonstrated one PTX-GO sheet heavily crumpled and self-roll itself at both edge and surface similar to other reports ${ }^{14}$. The drug loading content and drug loading efficiency in PTX-GO sheets were $89.2 \mu \mathrm{g} / \mathrm{mg}$ and $59.5 \%$, respectively.

In Raman spectra (Fig. 2), two dominant peaks appear at 1350 and $1600 \mathrm{~cm}^{-1}$ and are assigned to the D band and $G$ band of carbon atoms, respectively ${ }^{15}$. The $\mathrm{D}$ band presents the symmetric $\mathrm{A}_{\mathrm{g}}$ mode induced by edges, defects, dangling bonds and disorder carbon atoms, while the $\mathrm{G}$ band is the $\mathrm{E}_{2 \mathrm{~g}}$ mode presents the ordered $s p^{2}$-bonded carbon atoms. Therefore a higher $I_{D} / I_{G}$ ratio presents a higher level of disorder, which is an indicator of chemical functionalization in the carbon-based materials ${ }^{16}$. The $I_{D} / I_{G}$ ratio increased to 1.54 from 0.83 for pristine graphene oxide sheets, confirming the present of new $s p^{3}$ carbon atoms in paclitaxel-graphene oxide sheets induced by PTX-GO functionalizations.

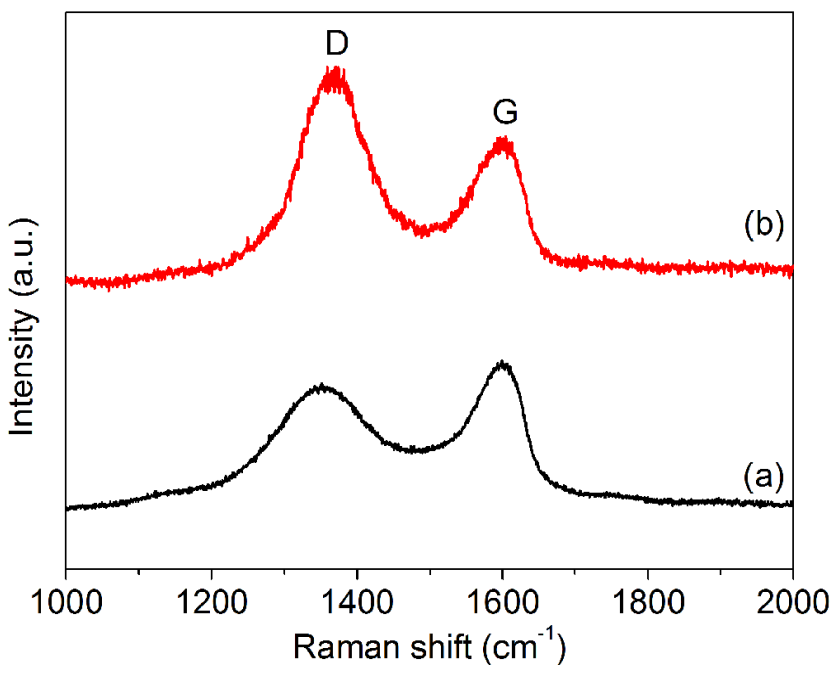

Fig. 2. Raman spectra of (a) graphene oxide sheets and (b) PTX-GO

Fig. 3A shows the electrosprayed PTX-GO nanospheres were uniform in size of 200-500 $\mathrm{nm}$. To have a deeper insight of the PTX-GO-gelatin nanospheres, a TEM image at a higher magnification in Fig. 3B shows their morphologies. The darker area in the core was expected to be assigned to the crumpled PTX-GO sheets resulted from their excellent flexibility. When a double amount $(20 \mathrm{mg})$ of PTX-GO sheets was introduced into gelatin solution, the electropraying process was also stable and fluent. Such electroprayed nanospheres were named as 
20-PTX-GO. The drug loading content values in 10-PTX-GO and 20-PTX-GO nanospheres were $14.9 \mu \mathrm{g} / \mathrm{mg}$ or $29.8 \mu \mathrm{g} / \mathrm{mg}$, respectively. Their drug loading efficiency kept the same value of $59.5 \%$, due to no loss of paclitaxel molecules during electrospraying.

Fig. 3C illustrates the in vitro releases of paclitaxel in PTXGO nanospheres. Both 10-PTX-GO and 20-PTX-GO nanospheres released approximately $20 \%$ of paclitaxel at the beginning $3 \mathrm{~h}$. But from then on, the 10-PTX-GO exhibited a lower release rate. At $36 \mathrm{~h}, 60 \%$ of paclitaxel released from (a) while $78.5 \%$ of paclitaxel released from (b). To clarify this smooth release mechanism, $7.34 \mathrm{mg}$ (the same amount of PTX-HPCD in $10 \mathrm{mg}$ of PTX-GO) was introduced into the gelatin solution yielding electrospray paclitaxel-gelatin nanospheres. Without the assistance of graphene oxide sheets, the PTX-HPCD molecules homogeneously dispersed in the gelatin nanospheres. And the PTX-HPCD transferred cross the gelatin nanospheres in the aqueous solution by permeation and diffusion, therefore paclitaxel molecules were quickly released by a dynamic process (Fig. 3C (c)). Furthermore, Fig. 3(d) showed that PTX-GO sheets released paclitaxel quickly from hydroxypropyl- $\beta$-cyclodextrin, although the PTX-HPCD had been strongly anchored on the graphene oxide sheets.
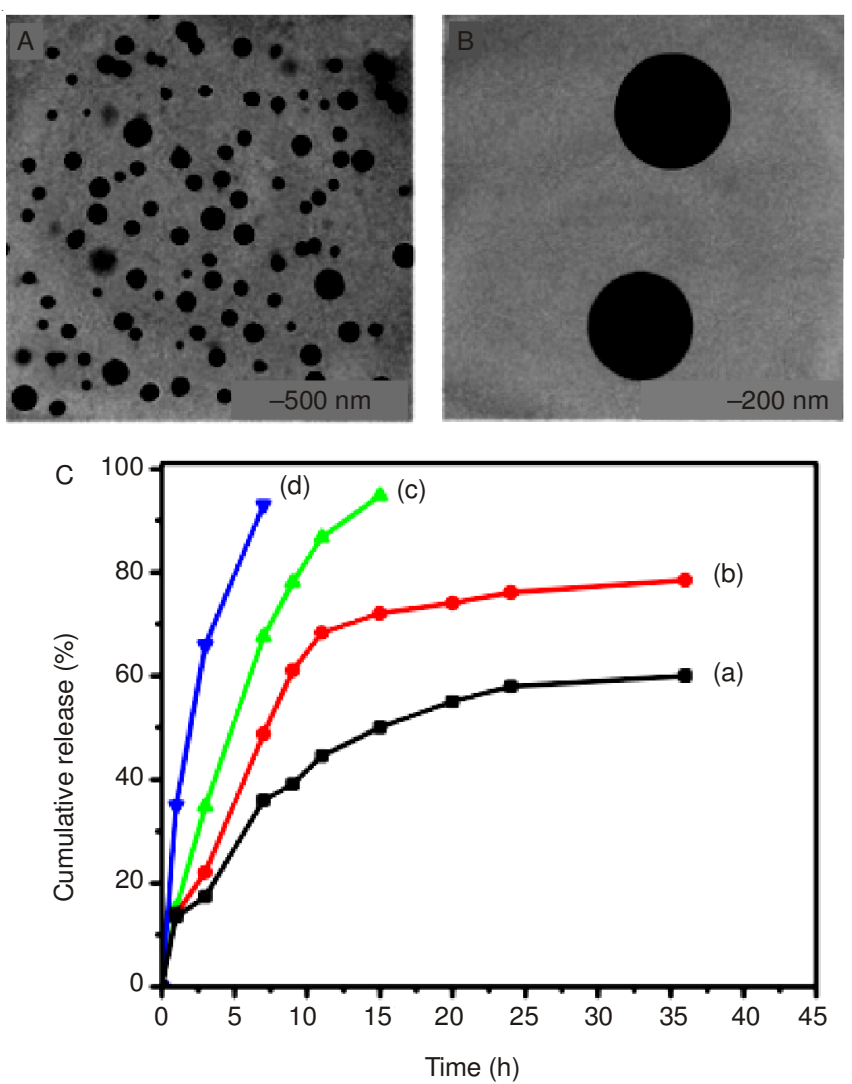

Fig. 3. (A and B) TEM images of paclitaxel-graphene oxide nanospheres (C) in vitro release profiles of PTX-GO nanospheres
That is, at the very beginning $1 \mathrm{~h}, 34.98 \%$ of paclitaxel was released from hydroxypropyl- $\beta$-cyclodextrin and this cumulative ratio reached to $65.93 \%$ at $3 \mathrm{~h}$ and $92.99 \%$ at $7 \mathrm{~h}$. Taken together, the graphene oxide nanospheres slowly and smoothly released paclitaxel, as a result of strong interaction between graphene oxide sheets and the hydroxypropyl- $\beta$ cyclodextrin molecules in addition to the encapsulation of graphene oxide nanospheres.

\section{Conclusion}

A new type of functionalized graphene oxide nanospheres has been developed by electrospraying technology. The functionalization of graphene oxide nanospheres by hydroxypropyl- $\beta$-cyclodextrin allowed themselves as successful anticancer drug carriers for unmodified paclitaxel. The drug loading concentration and drug loading efficiency in graphene oxide nanospheres were as high as $12.9 \mu \mathrm{g} / \mathrm{mg}$ and $59.5 \%$, respectively. The graphene oxide nanospheres exhibited slow and smooth in vitro release profile of paclitaxel. The strategy reported here is expected to be expandable to other waterinsoluble drugs, such as rifampin and/or indomethacin.

\section{ACKNOWLEDGEMENTS}

This work was financially supported by 973 Program (No. 2010CB732206 and No. 2012CB215306).

\section{REFERENCES}

1. J.M. Berlin, A.D. Leonard, T.T. Pham, D. Sano, D.C. Marcano, S. Yan, S. Fiorentino, Z.L. Milas, D.V. Kosynkin, B.K. Price, R.M. LucenteSchultz, X.X. Wen, M.G. Raso, S.L. Craig, H.T. Tran, J.N. Myers and J.M. Tour, ACS Nano, 4, 4621 (2010).

2. J.M. Berlin, T.T. Pham, D. Sano, K.A. Mohamedali, D.C. Marcano, J.N. Myers and J.M. Tour, ACS Nano, 5, 6643 (2011).

3. S. Li and M. Wang, Mater. Lett., 92, 350 (2013).

4. T.A. Hilder and J.M. Hill, Micro \& Nano Lett., 3, 41 (2008).

5. J. Zhang, Y. Liang, N. Li, X. Zhao, R. Hu, F. Hu, J. Xing, L. Deng and A. Dong, Micro \& Nano Lett., 7, 183 (2012).

6. Y. Jing, Y. Zhu, X. Yang, J. Shen and C. Li, Langmuir, 27, 1175 (2011).

7. X. Sun, Z. Liu, K. Welsher, J.T. Robinson, A. Goodwin, S. Zaric and H. Dai, Nano Res., 1, 203 (2008).

8. Z. Liu, J.T. Robinson, X. Sun and H. Dai, J. Am. Chem. Soc., 130, 10876 (2008).

9. A. Celebioglu and T. Uyar, Chem. Commun., 46, 6903 (2010).

10. K. Peng, I. Tomatsu, A.V. Korobko and A. Kros, Soft Matter, 6, 85 (2009).

11. W.S. Hummers Jr. and R.E. Offeman, J. Am. Chem. Soc., 80, 1339 (1958).

12. A.K. Geim and K.S. Novoselov, Nat. Mater., 6, 183 (2007).

13. S. Guo and S. Dong, Chem. Soc. Rev., 40, 2644 (2011).

14. C. Xu, J. Wang, L. Wan, J. Lin and X. Wang, J. Mater. Chem., 21, 10463 (2011).

15. K.N. Kudin, B. Ozbas, H.C. Schniepp, R.K. Prud'homme, I.A. Aksay and R. Car, Nano Lett., 8, 36 (2008).

16. J.M. Englert, C. Dotzer, G. Yang, M. Schmid, C. Papp, J.M. Gottfried, H.P. Steinrück, E. Spiecker, F. Hauke and A. Hirsch, Nat. Chem., 3, 279 (2011). 